\title{
POSITRON AFFINITIES AND DEFORMATION POTENTIALS IN CUBIC SEMICONDUCTORS
}

\author{
B.K. Panda and G. Brauer \\ Institut für Ionenstrahlphysik und Materialforschung, Forschungszentrum Rossendorf \\ Postfach 510119, 01314 Dresden, Germ : :y
}

\begin{abstract}
Positron affinities and deformation potentials are calculated in cubic bulk semiconductors using the density functional theory with the electron and positron energies in the local density approximation and generalized gradient approximation, respectively. In order to estimate these quantities, two different forms of the electron-positron correlation potential are used. Positron affinities calculated using these two correlation potentials differ by about $0.3 \mathrm{eV}$. Our calculated affinities in $3 \mathrm{C}-\mathrm{SiC}$ are in better agreement with experiments than those obtained previously by another first principles method. In the present work the positron affinity in $\mathrm{BN}$ is found to be quite close to the one in diamond.
\end{abstract}

PACS numbers: 79.20.Mb, 73.30.+y

\section{Introduction}

In the complete many-body theory of Lang and Kohn the electron work function $\left(\phi_{-}\right)$, which is the energy required for an electron to escape from the interior of the solid into vacuum, is separated into the bulk electron chemical potential $\left(\mu_{-}\right)$and the surface dipole potential $(\Delta)$ arising from the spilling out of the electrons beyond surface planes [1]. The work function is thus expressed as $\phi_{-}=-\mu_{-}+\Delta$. The electron cannot escape from the solid as it is electrically repelled back by the surface dipole potential. Since the positron is attracted by the surface dipole potential, the positron work function is expressed as $\phi_{+}=$ $-\mu_{+}-\Delta$. Measurements of the electron and positron work functions need clean sample surface as the dipole potential can be altered by absorbants. Puska et al. have defined the sum of electron and positron chemical potentials as the positron affinity $\left(A_{+}\right)[2]$ which is the relevant quantity in the comparison of the absolute positron energy levels in different materials. The positron affinity is related to the electron and positron work functions as

$$
A_{+}=-\left(\phi_{-}+\phi_{+}\right)=\mu_{-}+\mu_{+} \text {. }
$$


From this equation it is clear that the positron affinity is the bulk property of a homogeneous material. It is important in understanding the positron trapping at interfaces and precipitates. The positron affinity can be obtained from the positronium work function measurement [3]. However, the method is technically difficult and its precision not yet good enough to allow a reasonable comparison between theory and experiment. On the other hand, $A_{+}$of a material can be found from separate measurements of the electron and positron work functions provided the surface energy contributions are identical in both measurements [4]. However, the latter condition is very hard to achieve and $A_{+}$estimated in this way has got large scatter. The positron affinity can be measured in the positron emission spectroscopy method $[5,6]$. Weiss et al. have proposed that $A_{+}$can be measured from the kinetic edge of the positron induced secondary electron emission [7]. Since this experiment does not require the explicit emission of positrons from the sample, it can be used to measure $A_{+}$even in samples having positive positron work function.

The free positron diffusion in a semiconductor at low temperature is dominated by interaction with acoustic phonons. The contribution of positron acoustic phonon scattering is usually calculated using the Bardeen-Shockley formula which requires a precise value of the positron deformation potential [8]. The positron deformation potential $E_{\mathrm{d}}$ is obtained from the volume derivative of the positron affinity

$$
E_{\mathrm{d}}=\Omega \frac{\partial A_{+}}{\partial \Omega}
$$

where $\Omega$ is the volume of the crystal.

The estimation of the electron (positron) chemical potentials needs the electron (positron) ground state energies to be evaluated using a suitable common reference potential referred to the vacuum. In the Korringa-Kohn-Rostoker and augmented plane wave methods the common potential is calculated as the mean between the muffin-tin radius and Wigner-Seitz radius [9]. In the linear muffin-tin orbital within atomic spheres approximation (LMTO-ASA) method the Coulomb potential vanishes on the surface of the Wigner-Seitz cell. This allows the reference potential to be chosen uniquely at the surface of the Wigner-Seitz radius [2]. In the present work we have adopted the pseudopotential method where the average of the Coulomb potential is usually chosen as the reference potential [10].

\section{Theory}

The electron and positron states are calculated using the two-component density functional theory [11]. We adopted the simplified two-component density functional theory for the delocalised positron where there is only one positron in a many electrons system. In this scheme the evaluation of the electron states is independent of the positron density. The electron states are calculated in the pseudopotential method with the potential given by

$$
V_{-}(r)=V_{\mathrm{ps}}(r)+e^{2} \int \frac{n\left(r^{\prime}\right)}{\left|r-r^{\prime}\right|} \mathrm{d}^{3} r^{\prime}+V_{\mathrm{XC}}(r),
$$

where $V_{\mathrm{ps}}, n(r)$ and $V_{\mathrm{XC}}(r)$ are the pseudopotential of the ion, the valence pseudo-charge density and the exchange-correlation potential respectively. The second 
term in Eq. (3) is the repulsive Hartree potential. The pseudopotential $V_{\mathrm{ps}}(r)$ is given by the relation

$$
V_{\mathrm{ps}}(r)=\sum_{i, R}\left\langle l\left|V_{l}^{i}\left(r-R-\tau_{i}\right)\right| l\right\rangle
$$

where $R$ is the lattice position and $\tau_{i}$ is the position of the ion of $i$-th type. The summation over $i$ is carried out on all types of ions in the semiconductor. In our calculation we have employed the semi-local pseudopotential of the form [12]

$$
V_{l}^{i}(r)=-\frac{Z_{\mathrm{v}}^{i} e^{2}}{r} \operatorname{erf}\left(\sqrt{\alpha_{\mathrm{c}}^{i}} r\right)+\left(a_{l}^{i}+b_{l}^{i} r^{2}\right) \mathrm{e}^{-\alpha_{l}^{i} r^{2}},
$$

where $Z_{\mathrm{v}}^{i}$ is the valence charge of the $i$-th atom. The parameters $\alpha_{\mathrm{c}}, a_{l}, b_{l}$ and $\alpha_{l}$ are fitted to reproduce eigenvalues and wave functions of the all-electron methods. The exchange-correlation potential in Eq. (3) is obtained from the functional form of the exchange-correlation energy $\left(E_{\mathrm{XC}}[n]\right)$ as $V_{\mathrm{XC}}(r)=\delta E_{\mathrm{XC}}[n] / \delta n(r)$ where $E_{\mathrm{XC}}[n]$ in the local density approximation (LDA) is given by

$$
E_{\mathrm{XC}}[n]=\int n(r) \epsilon_{\mathrm{XC}}[n(r)] \mathrm{d}^{3} r .
$$

In order to obtain results consistent with the all-electron methods we have incorporated the nonlinear core correction (NLCC) technique [13] where $V_{\mathrm{XC}}$ is evaluated with the total pseudo-charge density $n_{0}(r)$ consisting of the pseudo-valence and pseudo-core charge densities.

In this work the Bachelet-Hamann-Schlüter pseudopotentials are used for all group IV and III-V semiconductors [14]. For the group II-VI semiconductors we have used the pseudopotentials of $\mathrm{Dal}$ Corso et al. [12]. The parametrized form of the exchange-correlation potential by Perdew and Zunger [15] is used in the present work. The chemical potential is calculated as

$$
\mu_{-}=E_{\mathrm{v}}+\alpha+V_{0}
$$

where $E_{\mathrm{v}}, \alpha$, and $V_{0}$ are the top of the valence band, the pseudo-core correction and the average of the Coulomb potential, respectively. Using Eq. (5) the pseudo-core correction is derived as

$$
\alpha=\frac{1}{\Omega} \int\left[\frac{Z_{\mathrm{v}} e^{2}}{r}-\frac{Z_{\mathrm{v}} e^{2}}{r} \operatorname{erf}\left(\sqrt{\alpha_{\mathrm{c}}} r\right)\right] \mathrm{d}^{3} r=\frac{2 \pi Z_{\mathrm{v}} e^{2}}{\Omega \alpha_{\mathrm{c}}} .
$$

The total positron potential contains the repulsive ion-core potential, the attractive Hartree potential and the electron-positron correlation potential. The ion-core potential is calculated in the point-core approximation. The correlation potential is evaluated in the generalized gradient approximation (GGA) where the potential depends on both the electron density and its gradient [16]. In order to reproduce results of the all electron potential method we have taken the NLCC in this case

$$
V_{+}(r)=\sum_{i, R} \frac{Z_{\mathrm{v}}^{i} e^{2}}{\left|r-R-\tau_{i}\right|}-e^{2} \int \frac{n\left(r^{\prime}\right)}{\left|r-r^{\prime}\right|} \mathrm{d}^{3} r^{\prime}+V_{\mathrm{corr}}^{\mathrm{GGA}}\left[n_{0}(r), \nabla n_{0}(r)\right] \text {. }
$$

Comparing Eq. (9) with Eq. (3) we find that the positron Hartree potential is the same in magnitude as the electron Hartree potential, but opposite in sign due to its charge. We have used the semi-empirical form of the correlation potential introduced by Barbiellini et al. [16]

$$
V_{\text {corr }}^{\mathrm{GGA}}\left[n_{0}(r), \nabla n_{0}(r)\right]=V_{\text {corr }}^{\mathrm{LDA}}\left[n_{0}(r)\right] \mathrm{e}^{-\beta \epsilon / 3},
$$


where $V_{\text {corr }}^{\mathrm{LDA}}$ is the correlation potential in LDA and $\epsilon$ is proportional to $\left|\nabla n_{0}\right|^{2} / n_{0}^{2}$. Here, $\beta$ is a parameter adjusted to the value of 0.22 .

The positron ground state energy is obtained as

$$
E_{0}=\int \Psi_{+}^{*}(r)\left[-\frac{\hbar^{2} \nabla^{2}}{2 m}+V_{+}(r)\right] \Psi_{+}(r) \mathrm{d}^{3} r
$$

where $\Psi_{+}$is the positron wave function. The positron chemical potential, calculated with respect to the average of the Coulomb potential, is given by

$$
\mu_{+}=E_{0}-V_{0} \text {. }
$$

We find that the calculation of the $\mu_{-}$and $\mu_{+}$using Eqs. (6) and (11) respectively, requires $V_{0}$ referred to the vacuum. In the pseudopotential method $V_{0}$ is calculated from the volume average of the Coulomb potential. Unfortunately, $V_{0}$ calculated in this manner is not unique and not referred to the vacuum [17]. However, using Eqs. (7) and (12) in Eq. (1) we find that the positron affinity does not require the explicit determination of $V_{0}$

$$
A_{+}=E_{\mathrm{v}}+E_{0}+\alpha \text {. }
$$

Unfortunately, up to now experimentally determined affinities do not exist for all semiconductors to check the accuracy of our calculations. However, measured positron lifetimes are available for most of the semiconductors and we can compare our calculations with them. The positron lifetime in the positron GGA scheme is given by the relation

$$
\lambda=\tau^{-1}=\pi r_{0}^{2} c \int n_{0}(r) n_{+}(r) \gamma_{\mathrm{GGA}}\left[n_{0}(r), \nabla n_{0}(r)\right] \mathrm{d}^{3} r .
$$

The enhancement factor within the GGA formalism [16] is given as

$$
\gamma_{\mathrm{GGA}}\left[n_{0}(r), \nabla n_{0}(r)\right]=1+\left\{\gamma_{\mathrm{LDA}}\left[n_{0}(r)\right]-1\right\} \exp (-\beta \epsilon)
$$

The significance of this semiempirical formula is that the enhancement factor approaches to that within the LDA formalism for the valence electrons $(\epsilon \approx 0)$ and becomes unity for the rapidly varying core electrons $(\epsilon \approx \infty)$.

\section{Results and discussion}

In the previous section it has been observed that the accuracy of the calculated positron affinities depends on the exact method of deriving electron and positron correlation energies. The correlation energies described in the LDA formalism are known to overbind atoms in a solid. It is assumed that this does not create any significant error in the present calculations as the experimental lattice constants were used for all semiconductors. The Perdew-Zunger parametrized form of the electron correlation potential is based on the quantum Monte Carlo (QMC) method and therefore it is widely accepted. However, there is no such parametrized form of the electron-positron correlation energy based on the QMC method. Here, the Boroński-Nieminen [11] and the recently proposed Boroński-Stachowiak [18] forms of the correlation energies have been used to compare positron affinities, deformation potentials and lifetimes - this is presented in Table.

It is found that lifetimes calculated using two different correlation energies are nearly the same, but affinities differ approximately by $0.3 \mathrm{eV}$ in all semiconductors. On the other hand, for samples with larger lattice constants, the deformation. 


\section{TABLE}

Positron affinity $A_{+}$, positron deformation potential $E_{\mathrm{d}}$ and lifetime $\tau$ calculated in the parametrized forms of the Boroński-Stachowiak (BS) and Boronski-Nieminen (BN) correlation energies in the local density approximation for differentsemiconductors. Experimental values of $\tau[16,21]$ are given for comparison.

\begin{tabular}{l|c|c|c|c|c|c|c}
\hline \hline & \multicolumn{3}{|c|}{ BS } & \multicolumn{3}{|c|}{ BN } & \begin{tabular}{c} 
Expt. \\
\cline { 2 - 6 }
\end{tabular} \\
\cline { 2 - 6 } & $\begin{array}{c}A_{+} \\
{[\mathrm{eV}]}\end{array}$ & $\begin{array}{c}E_{\mathrm{d}} \\
{[\mathrm{eV}]}\end{array}$ & $\begin{array}{c}\tau \\
{[\mathrm{ps}]}\end{array}$ & $\begin{array}{c}A_{+} \\
{[\mathrm{eV}]}\end{array}$ & $\begin{array}{c}E_{\mathrm{d}} \\
{[\mathrm{eV}]}\end{array}$ & $\begin{array}{c}\tau \\
{[\mathrm{ps}]}\end{array}$ & $\begin{array}{c}\tau \\
{[\mathrm{ps}]}\end{array}$ \\
\hline Diam & -2.67 & -11.48 & 101 & -2.30 & -11.79 & 101 & 100 \\
$\mathrm{Si}$ & -6.24 & -6.44 & 216 & -5.98 & -6.51 & 216 & 216 \\
$\mathrm{Ge}$ & -7.72 & -4.22 & 228 & -7.47 & -4.29 & 228 & 228 \\
$\mathrm{SiC}$ & -4.43 & -8.42 & 145 & -4.10 & -8.60 & 145 & 141 \\
$\mathrm{BN}$ & -3.78 & -10.33 & 109 & -3.37 & -10.60 & 109 & \\
$\mathrm{GaAs}$ & -7.90 & -4.71 & 225 & -7.64 & -4.78 & 225 & 231 \\
$\mathrm{GaP}$ & -7.95 & -4.91 & 219 & -7.69 & -4.98 & 219 & 221 \\
$\mathrm{CdTe}$ & -7.65 & -3.40 & 270 & -7.37 & -3.14 & 270 & 285
\end{tabular}

potentials are nearly the same in two correlation energies, but at those with smaller lattice constants the deformation potentials differ approximately by $0.3 \mathrm{eV}$. The calculated lifetimes are in good agreement with experiment for samples with low electron densities whereas for samples with high electron densities the agreement is poor. This is due to the fact that core electrons are not considered while constructing the positron ion-core potential.

The calculated positron affinity and deformation potential in diamond are found to be close to $-2.5 \mathrm{eV}$, a value given by another theoretical calculation [11]. Similarly, the calculated deformation potential is found to be slightly smaller than the earlier value of $-12.3 \mathrm{eV}$ obtained by the same method with a different crystal zero scheme [19].

In Si the affinity is smaller than $-6.95 \mathrm{eV}$ reported earlier by the LMTO-ASA method [2] whereas the deformation potential is closer to that calculation.

The positron affinity in $\mathrm{Ge}$ is found to have a higher value with the deformation potential being smaller than $2 \mathrm{eV}$ compared to LMTO-ASA results [11]. The cause for this discrepancy might be that the present method of calculation is based on the GGA scheme.

In $3 \mathrm{C}-\mathrm{SiC}$ the positron affinity is measured as $-3.83 \pm 0.45 \mathrm{eV}$ whereas an LMTO-ASA calculation with positron energies based on the GGA scheme estimates it to be $-5.61 \mathrm{eV}$ [20]. From Table I we find that the present calculation based on the Boronski-Nieminen formula for the correlation energy is in good agreement with the experiment.

Compared to other semiconductors we find that the positron affinity and deformation potential values of $\mathrm{BN}$ are quite similar to diamond. This shows that 
BN might be another candidate for the production of a field assisted moderator.

To our knowledge there are no theoretical and experimental results available in other semiconductors for comparison.

\section{Conclusions}

This is the first report of positron affinities and deformation potentials in compound semiconductors.

The electron exchange-correlation potential in the LDA method has been used to calculate these quantities. Further improvements are in progress to calculate the electron exchange-correlation potential in the GGA scheme which is supposed to reproduce structural properties of semiconductors. In addition, the frozen-core corrections for the top of the valence band will be taken into account to calculate positron affinities and deformation potentials.

One of us (B.K.P.) acknowledges the Alexander von Humboldt foundation for awarding a post-doctoral fellowship to work in Germany.

\section{References}

[1] N.D. Lang; W. Kohn, Phys. Rev. B 3, 1215 (1971).

[2] M.J. Puska, P. Lanki, R.M. Nieminen, J. Phys. Condens. Matter 1, 6081 (1989).

[3] R.H. Howell, I.J. Rosenberg, P. Meyer, M.J. Fluss, Phys. Rev. B 35, 4555 (1987).

[4] J. Störmer, A. Goodyear, W. Anwand, G. Brauer, P.G. Coleman, W. Triftshäuser, J. Phys., Condens. Matter 8, L89 (1996).

[5] D.W. Gidley, W.E. Frieze, Phys. Rev. Lett. 60, 1193 (1988).

[6] M. Jibaly, A. Weiss, A.R. Koymen, D. Mehl, L. Stiborek, C. Lei, Phys. Rev. B 44, 12166 (1991).

[7] A.H. Weiss, S.Yang, H.Q. Zhou, E. Jung, A.R. Koymen, S. Naidu, G. Brauer, M.J. Puska, Appl. Surf. Sci. 85, 82 (1995).

[8] J. Bardeen, W. Shockley, Phys. Rev. 80, 72 (1950).

[9] M. Weinert, R.E. Watson, Phys. Rev. B 29, 3001 (1984).

[10] C. Pennetta, Solid State Commun. 77, 159 (1991).

[11] M. Puska, R.M. Nieminen, Rev. Mod. Phys. 66, 841 (1994).

[12] A. Dal Corso, S. Baroni, R. Resta, S. de Gironcoli, Phys. Rev. B 47, 3588 (1993).

[13] S.G. Louie, S. Froyen, M.L. Cohen, Phys. Rev. B 26, 1738 (1982).

[14] G.B. Bachelet, D.R. Hamann, M. Schlüter, Phys. Rev. B 26, 4199 (1982).

[15] J. Perdew, A. Zunger, Phys. Rev. B 23, 5048 (1981).

[16] B. Barbiellini, M.J. Puska, T. Torsti, R.M. Nieminen, Phys. Rev. B 51, 7341 (1995).

[17] L. Kleinman, Phys. Rev. 80, 1532 (1981).

[18] E. Boroński, H. Stachowiak, Phys. Rev. B 57, 6215 (1998).

[19] B.K. Panda, C.D. Beling, S. Fung, Mater. Sci. Forum 175-178, 197 (1995).

[20] J. Kuriplach, M. Sob, G. Brauer, W. Anwand, E.-M. Nicht, P.G. Coleman, N. Wagner, Phys. Rev. B, in press.

[21] H. Siethoff, Phys. Status Solidi B 205, R3 (1998). 